\title{
Interference-Aware and Spectral-Efficient Resource Allocation Using Complete Graphs for the D2D Communication
}

\author{
Tsang-Ling Sheu, ${ }^{1}$ Yan-Jing Wu $\mathbb{D}^{2},{ }^{2}$ and Yen-Chieh Chiu ${ }^{1}$ \\ ${ }^{1}$ Department of Electrical Engineering, National Sun Yat-sen University, Kaohsiung, Taiwan \\ ${ }^{2}$ Department of Information Technology and Communication, Shih Chien University, Kaohsiung Campus, Kaohsiung, Taiwan \\ Correspondence should be addressed to Yan-Jing Wu; yanjing0622@gmail.com
}

Received 13 December 2021; Revised 22 January 2022; Accepted 7 February 2022; Published 28 February 2022

Academic Editor: Issa Elfergani

\begin{abstract}
Copyright (c) 2022 Tsang-Ling Sheu et al. This is an open access article distributed under the Creative Commons Attribution License, which permits unrestricted use, distribution, and reproduction in any medium, provided the original work is properly cited.
\end{abstract}

\begin{abstract}
This paper proposes a heuristic resource block allocation using complete graphs (abbreviated to RACG) mechanism for device-todevice (D2D) communication. RACG not only can reduce the cochannel interference but also can improve the overall system capacity. For RACG, we define two pieces of user equipment in the D2D communication as one D2D pair and use the interference between each D2D pair to establish an interference graph model, which is then used to identify all complete graphs with different numbers of vertices. The innovation of RACG is right that it begins with resource block (RB) allocation for the D2D pairs in the complete graph with the largest number of vertices and considers three additional parameters to determine the allocation sequence for the $\mathrm{D} 2 \mathrm{D}$ pairs. The three additional parameters are the frequency at which $\mathrm{D} 2 \mathrm{D}$ pairs appear in complete graphs, the interference level of a D2D pair to other D2D pairs, and the number of RBs required. Furthermore, RACG prioritizes the reuse of allocated RB chunks during RB allocation. When a D2D pair and other D2D pairs with allocated RBs do not have mutual interference, the allocated RB chunk that has the closest number of RBs to the number of RBs required is selected and reused. We conduct performance analysis and compare the proposed RACG with a resource allocation of degree-based greedy (RADG) approach. The performance measures include the RB usage rate and occupied rate in a frame.
\end{abstract}

\section{Introduction}

The development of mobile communication technology brings about massive increases of user equipment (UE) such as smart phones, machine-to-machine modules, and tablets, which in turn cause sudden increases in bandwidth demand in mobile communication networks. UE do not even have sufficient bandwidth for transmission during peak hours or in cities with a high population density, resulting in intolerable packet delay. To provide sufficient bandwidth to an increasing number of UE and maintain low packet delay, the Third-Generation Partnership Project (3GPP) has proposed a new device-to-device (D2D) communication mode for new generation mobile communication networks $[1,2]$. We define two pieces of UE in the D2D communication as one $\mathrm{D} 2 \mathrm{D}$ pair. The $\mathrm{D} 2 \mathrm{D}$ communication can reduce the transmission power and, consequently, the interference pro- duced by the signal. Therefore, the D2D pairs that do not have mutually overlapping transmission ranges can use the same bandwidth resource to transmit simultaneously; through frequency reuse, the overall system capacity can be enhanced.

Figure 1 illustrates the medium access technology applied in 4G and beyond, namely, Orthogonal Frequency Division Multiple Access (OFDMA) [3]. An OFDMA frame consists of both frequency domain and time domain. The frequency domain is measured in Hertz $(\mathrm{Hz})$ and consists of multiple carriers; each carrier is $180 \mathrm{KHz}$ and can be split into 12 subcarriers. Therefore, each subcarrier is $15 \mathrm{KHz}$. The time duration of an OFDMA frame is $10 \mathrm{~ms}$, and each frame is divided into ten subframes. Hence, each subframe is $1 \mathrm{~ms}$. A subframe consists of two subslots, where each subslot is $0.5 \mathrm{~ms}$ and consists of seven symbols. In the 4G LongTerm Evolution-Advanced (LTE-A) standard, the smallest 


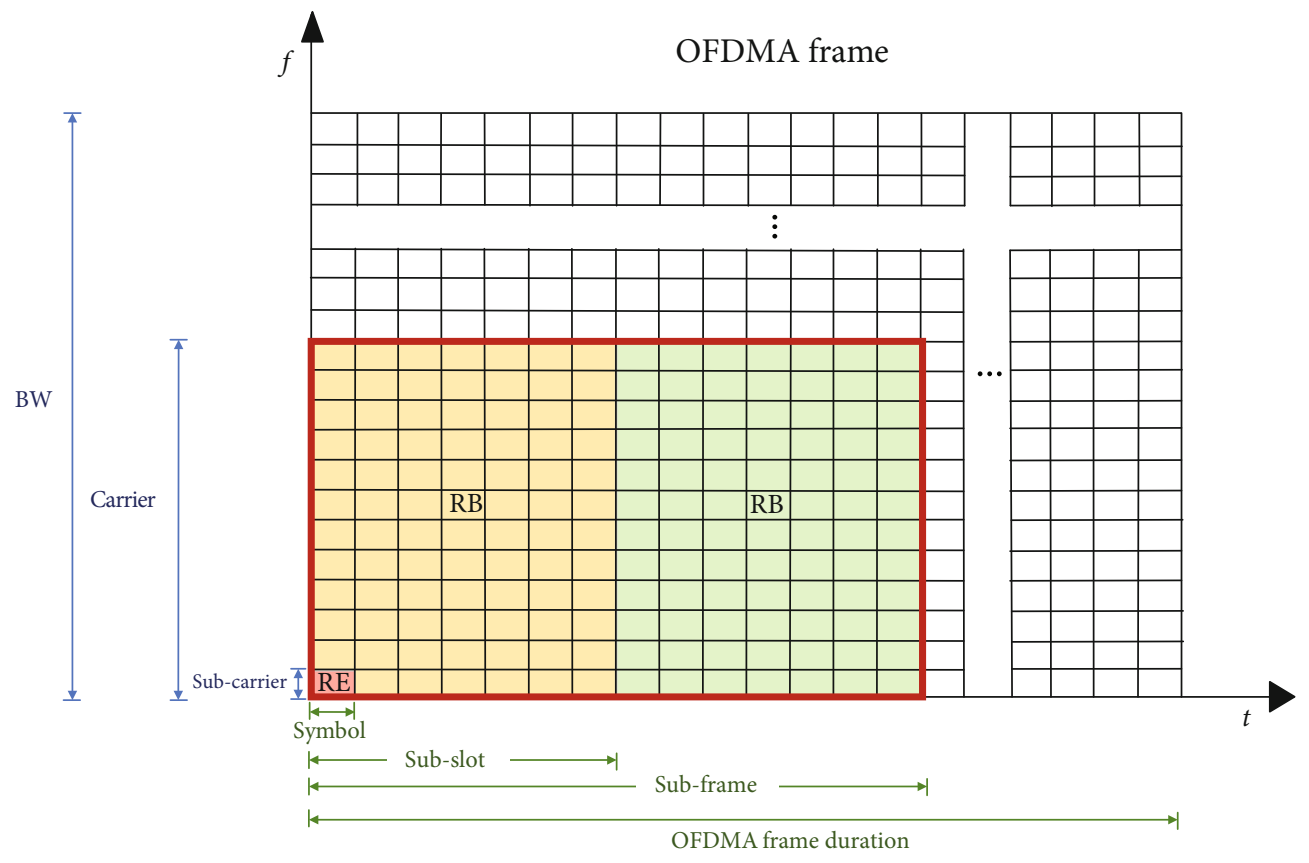

FIgURE 1: Framework of OFDMA.

resource element (RE) and smallest resource block (RB) are defined. One RE consists of one symbol and one subcarrier, whereas one RB consists of seven symbols and one carrier. Therefore, each RB has 84 REs. Since the bandwidth requirement for one piece of UE can be one or more RBs, the scheduler at eNodeB must integrate multiple RBs and allocate them to the UE. This paper defines the bandwidth provided by the integration of multiple RBs as one RB chunk. For example, the RB chunk enclosed by the bold red line in Figure 1 is composed of two RBs.

Figure 2 demonstrates that one D2D pair consists of one sender and one receiver. In 3GPP Release13, the direct communication channel between a sender and a receiver is designated as a sidelink [4]. When the sender needs to transmit data, it uses a Sidelink Buffer Status Report (S-BSR) packet to notify the eNodeB about its bandwidth requirement. The eNodeB then allocates an adequate RB chunk to the sidelink according to the received S-BSR packet and informs the sender via a Physical Downlink Control Channel (PDCCH). Before the commencement of the D2D communication, the sender uses a Physical Sidelink Control Channel (PSCCH) to send information to the receiver; this information relates to the RB chunk allocated to the sidelink.

Because the transmission power of the D2D communication is small, it is highly probable that the transmission ranges of $\mathrm{D} 2 \mathrm{D}$ pairs do not overlap with one another. The D2D pairs that do not have overlapping transmission ranges can reuse the same bandwidth resource $[5,6]$ to enhance the overall system capacity. Figure 3 illustrates that each D2D pair comprises one sender and one receiver; the senders are marked in gold (e.g., DUE 1, DUE 3, DUE 5, and DUE $n-1)$, whereas the receivers are marked in gray (e.g., DUE 2, DUE 4, DUE 6, and DUE $n$ ). In Figure 3, the D2D pairs covered with different colors stand for communication via different RB chunks. The bottom section of Figure 3 shows two allocated RB chunks in one OFDMA frame, where one is marked in green and the other in blue. These two $R B$ chunks do not reuse the same RBs. The eNodeB cell coverage in Figure 3 contains $m$ D2D pairs. Because the transmission ranges of the D2D pairs may mutually interfere with each other (e.g., D2D pairs 1 and 2), D2D pairs 1 and 2 use the blue and green RB chunks, respectively. However, D2D pairs 2, 3, and $m$ can use the same green RB chunk because their individual transmission ranges do not mutually interfere with each other.

Without D2D communication, packet exchange between one pair of UE needs the forwarding of eNodeB, resulting in the waste of bandwidth resource and the increase of packet delay. The D2D direct communication reduces the transmission power of UE and cochannel interference. Therefore, RBs can be reused repeatedly, and this repeated usage can enhance the overall system capacity. Aiming to maximize the benefits of frequency reuse and reduce the interference level among adjacent D2D pairs, this paper proposes a heuristic $\mathrm{RB}$ allocation mechanism using complete graphs (abbreviated to RACG). At first, RACG establishes an interference graph model and identifies all complete graphs with different numbers of $\mathrm{D} 2 \mathrm{D}$ pairs in the model. The main contribution of RACG is that it begins with RB allocation for the D2D pairs in the complete graph with the largest number of D2D pairs and takes into account three additional parameters to determine the allocation sequence for the D2D pairs. The three additional parameters are the frequency at which D2D pairs appear in complete graphs, the interference level of a D2D pair to other D2D pairs, and the number of RBs required. Furthermore, RACG prioritizes the reuse of allocated $\mathrm{RB}$ chunks during $\mathrm{RB}$ allocation. When a D2D pair and other D2D pairs with allocated RBs do not have mutual 


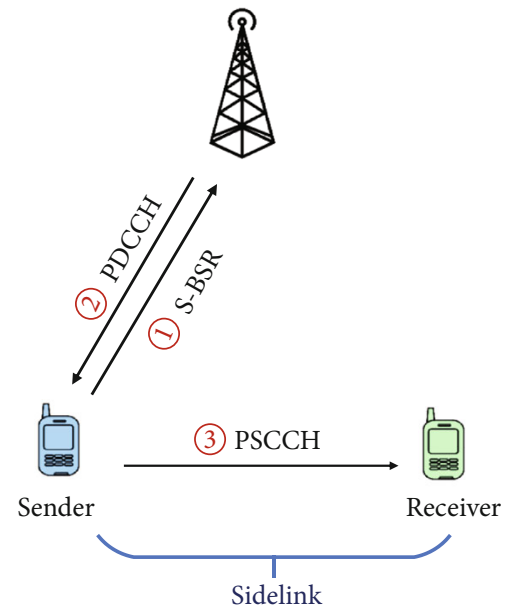

FIgURE 2: Operating procedure of D2D communication.

interference, the allocated $\mathrm{RB}$ chunk that has the closest number of RBs to the number of RBs required is selected and reused. In order to demonstrate the advantages of the proposed RACG, we design two topologies that represent two types of D2D pair distribution, namely, hotspot and random topologies. For the hotspot topology, we select a fixed number of D2D pairs and change the level of interference among the D2D pairs near the hotspot. For the random topology, we gradually increase the total number of D2D pairs. The performance measures include the $\mathrm{RB}$ usage rate and occupied rate in an OFDMA frame.

The remainder of this paper is organized as follows. Section 2 explores the related literature. Section 3 describes the operating procedures of the proposed RACG. Section 4 analyzes and compares the numerical results. Finally, concluding remarks and future works are given in Section 5.

\section{Related Works}

In recent years, studies have proposed $\mathrm{RB}$ allocation schemes under the D2D communication. These studies can be classified into four types. The first type of studies [7-12] clustered the D2D pairs based on UE behavior in social networks and applied the game theory to determine optimal RB allocation. The second type of studies [13-18] collected actual geographical locations of D2D pairs to identify the D2D pairs that had no mutual interference and enabled these D2D pairs to reuse the same RB chunk. The third type of studies [19-24] proposed an RB allocation principle with Quality of Services (QoS) provision. The transmission power of each D2D pair was calculated to identify the level of mutual interference, and RB chunks were then allocated to the $\mathrm{D} 2 \mathrm{D}$ pairs according to their respective QoS requirements. The fourth type of studies [25-31] created an interference graph model to represent the interference between D2D pairs. As illustrated in Figure 4, each vertex represents a D2D pair. If two D2D pairs mutually interfere with each other, an edge is used to connect two vertices together. Therefore, the degree of each vertex represents the level of interference corresponding to the D2D pair. Each vertex is colored while ensuring that its neighboring vertices do not have the same color. Vertices with the same color are allocated with the same RB chunk to increase the benefits of frequency reuse.

The fourth type of studies is more closely related with the RACG proposed in this paper, and a summary of these studies is provided in this section. Lee et al. [25] proposed an $\mathrm{RB}$ allocation with a degree-based greedy (RADG) approach. RADG first calculates the degree of each vertex in an interference graph; next, it selects the D2D pair that has the highest degree (i.e., the D2D pair that causes the largest amount of interference) to perform $\mathrm{RB}$ allocation first. If two or more vertices have the same degree, RADG then prioritizes $\mathrm{RB}$ allocation for the $\mathrm{D} 2 \mathrm{D}$ pair with the larger bandwidth requirement. When the bandwidth requirements are the same, RADG then randomly selects a D2D pair to allocate RBs. RADG is degree-based and does not consider the mutual interference among multiple D2D pairs. Therefore, some D2D pairs cannot reuse the allocated $\mathrm{RBs}$, which reduces the benefits of frequency reuse. In a full duplex environment, T. Yang et al. [26] focused on determining the appropriate transmission powers for uplink, downlink, and D2D communication by establishing the interference graphs of different links according to the bandwidth requirement and interference level for each D2D pair. Li et al. [27] proposed a joint mode selection and resource allocation algorithm by dividing the users into groups via the vertex coloring approach. After solving the power optimization problem for each group and conducting mode selection between the cellular mode and D2D mode for each D2D pair, they assigned channel resources to these groups. On the other hand, the studies in [28-31] considered an environment in which cellular UE and D2D pairs coexist. The authors of $[28,29]$ considered the level of interference between cellular UE and D2D pairs, and they established an interference graph to determine the optimal RB allocation for achieving the coexistence of cellular UE and D2D pairs. However, cellular UE have higher priority, and an excessive number of cellular UE can cause the starvation of the D2D pairs. Zhao et al. [30] proposed an algorithm that uses an interference graph to decide an RB allocation model. If the interference level of a D2D pair is smaller than a predefined threshold, the D2D pair can reuse the RBs that have allocated to cellular UE. Otherwise, new RBs are allocated to the D2D pair. Without considering the mutual interference between multiple $\mathrm{D} 2 \mathrm{D}$ pairs, the proposed $\mathrm{RB}$ allocation algorithm is apt to allocate new RBs to some D2D pairs, and this can lead to insufficient system bandwidth. Cai et al. [31] assumed that the number of RBs required by all of cellular UE and D2D pairs is identical, and they used graph coloring to identify the RBs that can be reused. Next, they used the signal-to-interference-plus-noise ratio (SINR) to calculate the transmission rate of these reusable RBs and allocated the RBs with the highest transmission rate to the corresponding D2D pair to meet the bandwidth requirement of each D2D pair. However, this also resulted in some D2D pairs not having reusable RBs, which reduced the benefits of frequency reuse. The application of graph coloring for the above-mentioned RB allocation schemes may result in uneven RB allocation between cellular UE and D2D pairs. 


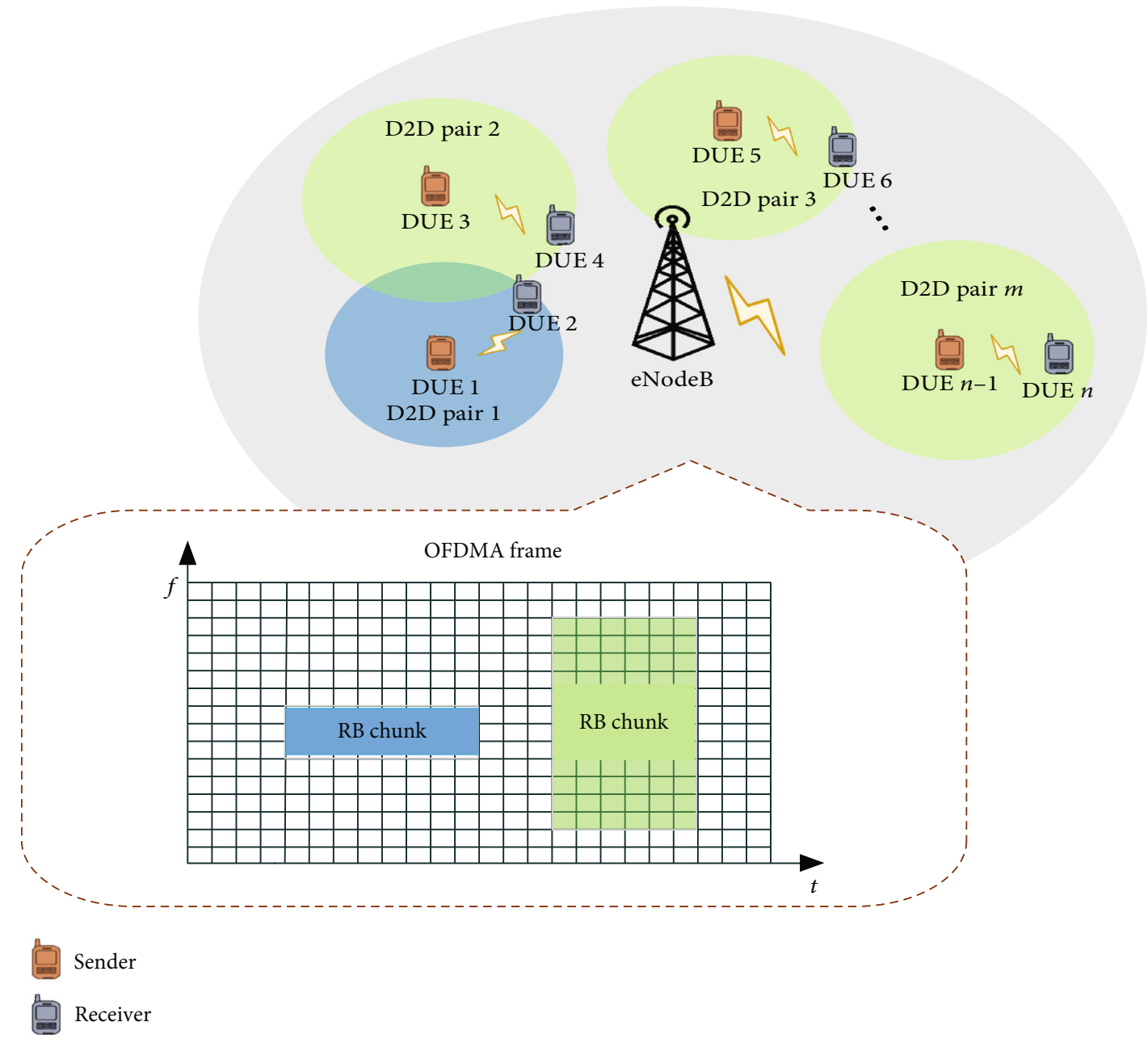

FIgURE 3: Frequency reuse of D2D communication.

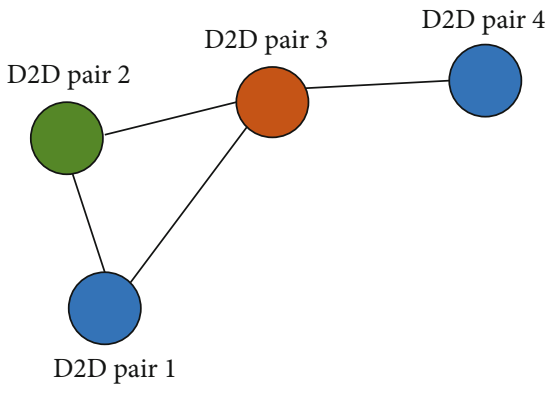

Figure 4: Interference graph model.

In addition, some applications ignore the level of interference between multiple D2D pairs, which leads to fewer reusable RBs because of limited bandwidth resource and therefore affects the overall system capacity.

Aiming to maximize the benefits of frequency reuse and reduce the level of interference among adjacent D2D pairs, this paper proposes a heuristic RACG. RACG first maps the interference between D2D pairs to an interference graph model, after which it applies the Bron-Kerbosch algorithm [32] to identify every complete graph within the interference graph. A complete graph (denoted as $K_{n}$ ) is an undirected graph with $n$ vertices, and any two different vertices have exactly one edge connecting them [33]. RACG begins with $\mathrm{RB}$ allocation for $\mathrm{D} 2 \mathrm{D}$ pairs in the complete graph with the highest number of vertices. The allocation sequence for $\mathrm{D} 2 \mathrm{D}$ pairs is determined according to three additional parameters, namely, the number of times D2D pairs appear in different complete graphs, the interference effect of a D2D pair on other D2D pairs, and the number of $\mathrm{RBs}$ required by a D2D pair. Besides, RACG prioritizes the reuse of RB chunks during $\mathrm{RB}$ allocation. In other words, if a D2D pair and other D2D pairs allocated with RBs do not mutually interfere with each other, we select the allocated RB chunk with the closest number of RBs relative to the number of $\mathrm{RBs}$ required. If the $\mathrm{RB}$ requirement of this $\mathrm{D} 2 \mathrm{D}$ pair exceeds all allocated RB chunks, RACG reuses the largest $\mathrm{RB}$ chunk and adds unallocated RBs.

\section{The Proposed RACG}

3.1. Interference Graph Model. The RACG mechanism first maps the interference between all D2D pairs to an interference graph model. In the model, each vertex represents 
one $\mathrm{D} 2 \mathrm{D}$ pair, and an edge connecting two vertices indicates the mutual interference between two D2D pairs. $V_{n}$ and $E_{n}$, respectively, denote a vertex set and edge set in an interference graph. Let $V_{n}=\left\{v_{1}, v_{2}, \cdots, v_{n}\right\}$, and the adjacency matrix $A$ is defined to represent the interference graph. $A$ is a symmetric matrix and its size is $n \times n$. The elements of $A$ are $a_{i, j}$ (Equation (1)). When $a_{i, j}=1$, the transmission ranges of the $i$ th and $j$ th vertices overlap. Conversely, when $a_{i, j}=0$, the transmission ranges of the two vertices do not overlap. The edge set is $E_{n}=\left\{e_{i, j} \mid \forall v_{i}, v_{j} \in V_{n}, i \neq j, a_{i, j}=1\right\}$. The symbol $G_{n}$ is used to represent the interference graph whose number of D2D pairs is $n$, and $G_{n}=\left(V_{n}, E_{n}\right)$. For example, Figure 5 shows an interference graph of six D2D pairs, and the adjacency matrix of $G_{6}$ is demonstrated in Equation (2). In the interference graph of Figure 5, the vertex set $V_{6}$ is $\left\{v_{1}, v_{2}, v_{3}, v_{4}, v_{5}, v_{6}\right\}$, and the edge set $E_{6}$ is $\left\{e_{12}, e_{13}, e_{23}, e_{34}, e_{45}, e_{56}\right\}$.

$$
\begin{aligned}
& a_{i j}=\left\{\begin{array}{cc}
1, & \text { if } v_{i} \text { and } v_{j} \text { are adjacent } \\
0, & \text { otherwise }
\end{array}\right\} \\
& \in A, \forall 1 \leq i \leq n, \forall 1 \leq j \leq n \text {, }
\end{aligned}
$$

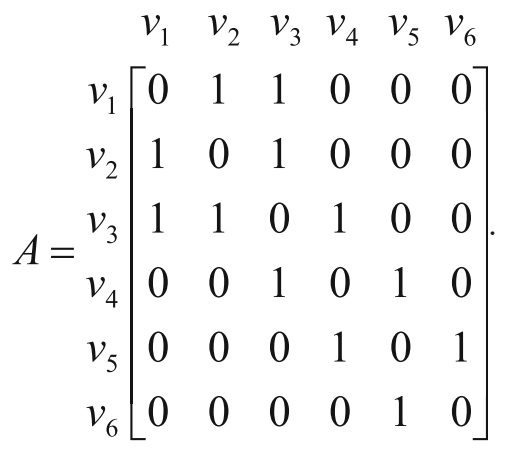

3.2. RB Allocation Approach. RACG identifies all complete graphs with different number of vertices in the interference graph. Thereafter, it commences RB allocation for the D2D pairs in the complete graph with the largest number of vertices. Different RB chunks are allocated such that the number of $\mathrm{RB}$ chunks which can be reused by other D2D pairs increases. These principles allow more D2D pairs to use the same RBs with limited bandwidth resources and increase the benefits of reusing resources. Some D2D pairs may belong to two or more complete graphs with identical sizes. In other words, the vertices of these D2D pairs belong to the intersection of multiple complete graphs with identical sizes. As shown in Figure 6, the interference graph has the two largest complete $K_{4}$ graphs, namely, $\left\{v_{1}, v_{2}, v_{3}, v_{4}\right\}$ and $\left\{v_{2}, v_{4}, v_{5}, v_{6}\right\}$, and the intersection between these two $K_{4}$ graphs is $\left\{v_{2}, v_{4}\right\} . v_{2}$ and $v_{4}$ are allocated RB chunks which do not overlap prior to the other vertices for the purpose of reducing the level of interference among adjacent $\mathrm{D} 2 \mathrm{D}$ pairs. $\mathrm{RB}$ allocation starts with the $\mathrm{D} 2 \mathrm{D}$ pairs in the compete graph that has the largest number of vertices, and three parameters are used to determine the allocation sequence of D2D pairs. The three parameters are the number of times D2D pairs appear in different complete graphs, the degree to which a

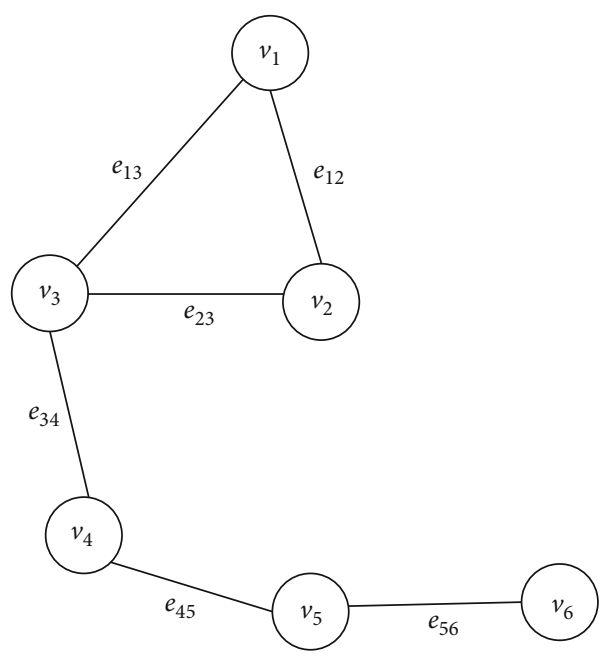

Figure 5: Interference graph of six D2D pairs.

D2D pair interferes with other D2D pairs, and the number of $\mathrm{RBs}$ required by a $\mathrm{D} 2 \mathrm{D}$ pair. $\mathrm{RB}$ allocation to the $\mathrm{D} 2 \mathrm{D}$ pairs of $K_{3}$ will start after RB allocation to the D2D pairs of $K_{4}$ has finished. RACG prioritizes the allocation of reusable $\mathrm{RB}$ chunks. That is, if a D2D pair and other D2D pairs allocated with RBs do not mutually interfere with each other, RACG selects and reuses an allocated RB chunk that has the closest number of RBs to the number of RBs required. Once the $\mathrm{RB}$ requirement of a $\mathrm{D} 2 \mathrm{D}$ pair exceeds all allocated $\mathrm{RB}$ chunks, RACG will reuse the largest RB chunk and adds unallocated RBs.

Table 1 lists the parameters used by RACG and their corresponding definitions. $n$ is the number of all vertices in the interference graph. $V_{m, j}$ is the vertex set of the $j$ th $K_{m}$ complete graph. $M K_{m}$ is the matrix with a size of $n \times t_{m}$, which is made of all $K_{m}$ complete graphs. $t_{m}$ is the total number of $K_{m}$ complete graphs in the interference graph. $c_{i, k}$ denotes the element in the $i$ th row and $k$ th column of $M K_{m} ; c_{i, k}$ is either 0 or 1 , as demonstrated in Equation (3). If $c_{i, k}$ is equal to 1 , then vertex $v_{i}$ belongs to $V_{m, k}$; otherwise, $c_{i, k}$ is equal to 0 . As expressed by Equation (4), $f_{m, i}$ denotes the sum of all elements in the $i$ th row of $M K_{m}$, and it indicates the number of $K_{m}$ complete graphs that vertex $v_{i}$ belongs to. $r b v_{i}$ and $d v_{i}$ represent, respectively, the requirement of RBs and degree of vertex $v_{i}$.

$$
\begin{gathered}
c_{i, k}= \begin{cases}1, & \text { if } v_{i} \in V_{m, k} \\
0, & \text { otherwise }\end{cases} \\
f_{m, i}=\sum_{k=1}^{t_{m}} c_{i, k} .
\end{gathered}
$$

The interference graph of Figure 7 is presented as an example. Through applying the Bron-Kerbosch algorithm (mentioned in Section 2), six complete graphs are identified in Figure 7. The vertex sets of these complete graphs are $V_{4,1}=\left\{v_{1}, v_{2}, v_{3}, v_{4}\right\}, V_{4,2}=\left\{v_{3}, v_{4}, v_{5}, v_{6}\right\}, \quad V_{4,3}=\left\{v_{1}, v_{2}\right.$, $\left.v_{3}, v_{10}\right\}, V_{3,1}=\left\{v_{4}, v_{6}, v_{8}\right\}, V_{3,2}=\left\{v_{6}, v_{7}, v_{8}\right\}$, and $V_{2,1}=$ 


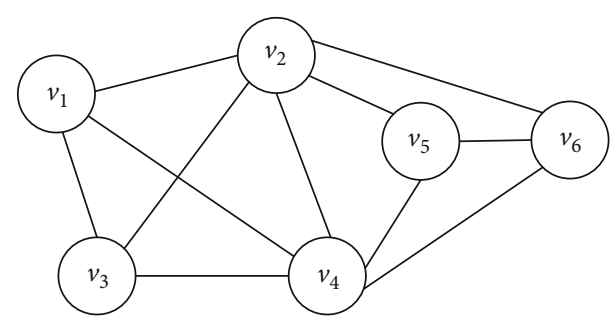

FIGURE 6: Interference graph with vertices belonging to multiple complete graphs.

TABLE 1: Parameters and definitions.

\begin{tabular}{lc}
\hline Parameter & Definition \\
\hline$n$ & The number of all vertices \\
$V_{m, j}$ & Vertex set of $j$ th $K_{m}$ complete graph \\
$M K_{m}$ & Matrix formed by all $K_{m}$ complete graphs \\
$c_{i, k}$ & Element in the ith row and $k$ th column of $M K_{m}$ \\
$f_{m, i}$ & Sum of all elements in ith row of $M K_{m}$ \\
$r b v_{i}$ & Number of RBs required by vertex $v_{i}$ \\
$d v_{i}$ & Degree of vertex $v_{i}$ \\
\hline
\end{tabular}

$\left\{v_{8}, v_{9}\right\}$. Therefore, the largest complete graph is $K_{4}$, and three $K_{4}$ s are identified. The size of $M K_{4}$ is $10 \times 3$, and the value of each element is as shown in Equation (5). Next, the calculation indicates that $f_{4,1}=2, f_{4,2}=2, f_{4,3}=3, f_{4,4}=2$, $f_{4,5}=1, f_{4,6}=1, f_{4,7}=0, f_{4,8}=0, f_{4,9}=0$, and $f_{4,10}=1$. Therefore, $v_{3}$ has the highest level of interference, and RBs are first allocated to the $\mathrm{D} 2 \mathrm{D}$ pair that $v_{3}$ represents. The vertices $v_{1}$, $v_{2}$, and $v_{4}$ have the same level of interference; hence, RBs are first allocated to the $\mathrm{D} 2 \mathrm{D}$ pair that $v_{4}$ represents because the degree of $v_{4}$ (denoted as $d v_{4}$ ) is greater than $d v_{1}$ and $d v_{2}$. Because the degrees of $v_{1}$ and $v_{2}$ are both four, the sequence of $\mathrm{RB}$ allocation is determined by the $\mathrm{RB}$ requirement of the D2D pairs represented by the two vertices (denoted as $r b v_{1}$ and $r b v_{2}$ ).

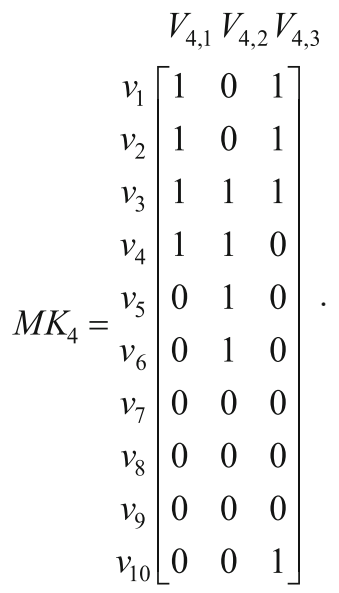

3.3. Pseudocode of RACG. Pseudocode 1 presents the pseudocode of RACG. First, as discussed in Section 3.1, RACG establishes the interference graph model, $G_{n}=\left(V_{n}, E_{n}\right)$, of

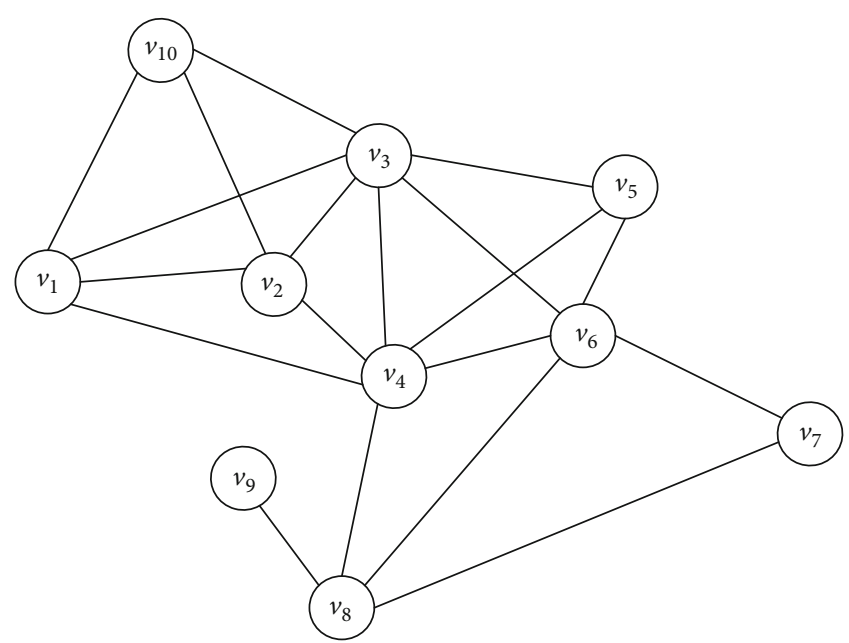

FIGURE 7: Interference graph with multiple complete graphs of varying sizes.

D2D communication. Next, it applies the Bron-Kerbosch algorithm to identify all complete graphs in the interference graph and obtains $M$ vertices in the largest complete graph. Thereafter, $M K_{1}, M K_{2}, \ldots, M K_{M}$ are established, and $f_{m, i}$, of each vertex $i$ is calculated $(1 \leq m \leq M)$. Per the first principle of RACG, RBs are allocated to the vertex with the largest $f_{M, i}$ (i.e., D2D pair $i$ ). When two or more vertices have the same $f_{M, i}$, the second principle of RACG is applied; that is, these vertices are ranked from largest to smallest according to their degree number for RB allocation. When the degree number of the vertices are the same, the third principle of RACG is applied; that is, RBs are allocated to the vertex that represents the D2D pair requiring the largest number of RBs. When the number of RBs required is identical, a vertex for $\mathrm{RB}$ allocation is selected at random.

During RB allocation, when D2D pair $i$ is the first to be allocated, then the required RBs $\left(r b v_{i}\right)$ are directly allocated to it. By contrast, when this D2D pair and other D2D pairs allocated with RBs mutually interfered with each other, this D2D pair do not reuse the allocated RBs and new RBs will be allocated to it. When this D2D pair and other D2D pairs allocated with RBs do not mutually interfere with each other, the following rules are applied to reuse the allocated $R B$ chunks. (1) The RB chunk that is greater than or equal to $r$ $b v_{i}$ and closest in value to $r b v_{i}$ is reused; and (2) the RB chunk that is smaller than $r b v_{i}$ and closest in value to $r b v_{i}$ is reused, and unallocated RBs are added to meet the RB requirement of $v_{i}$.

\section{Performance Evaluation}

4.1. Simulation Scenarios. We design two network topologies of D2D communication in LTE-A systems as shown in Figure 8 . The blue circle represents the cell coverage of an eNodeB, and two pieces of UE form a single D2D pair. The distribution of all D2D pairs in Figure 8(a) represents a hotspot topology. Hence, most D2D pairs are concentrated around the eNodeB with only a few D2D pairs being 


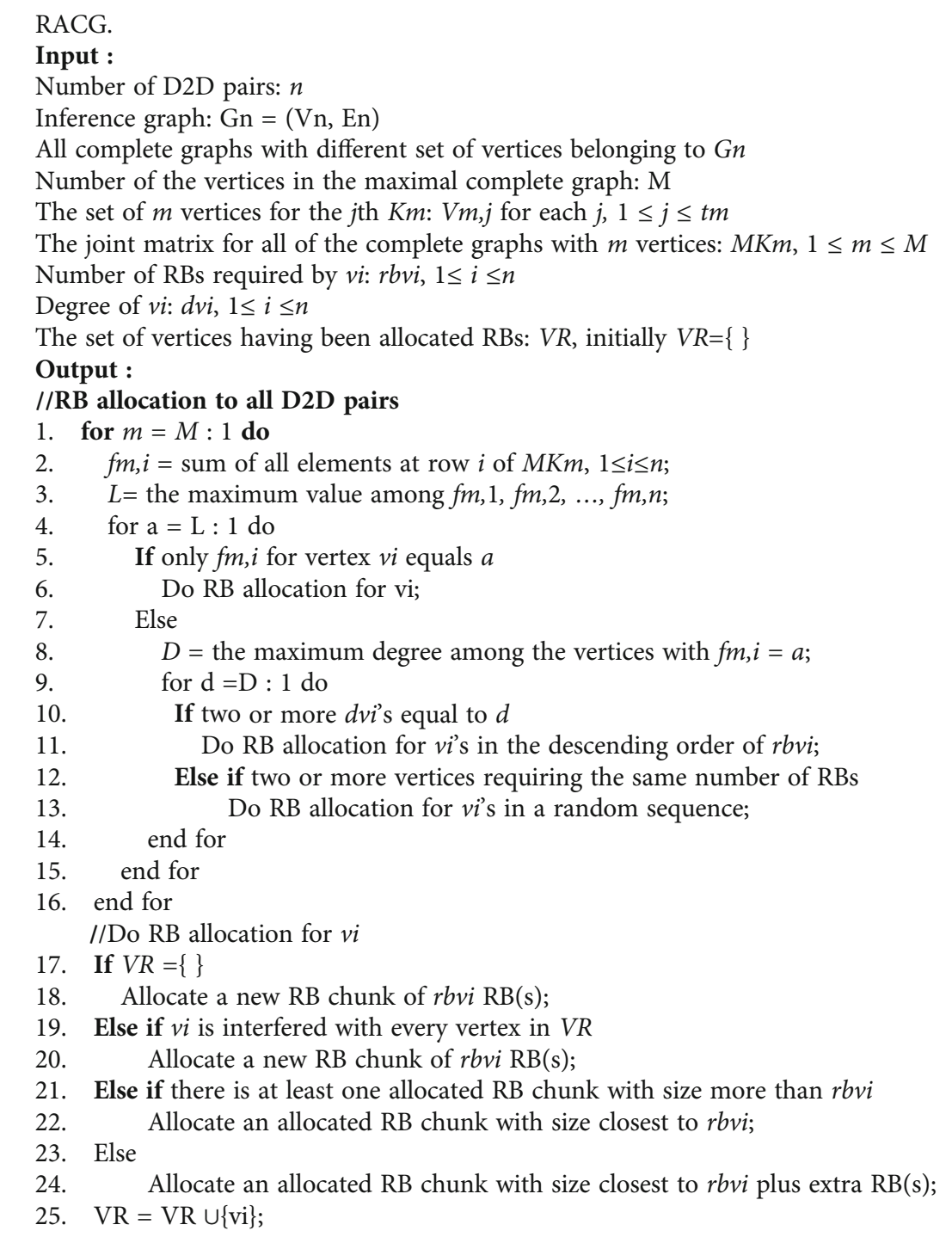

Pseudocode 1

exceptions. Figure 8(b) represents randomly distributed D2D pairs in a random topology.

We use MATLAB [34] to perform a numerical simulation in which the total number of RBs of an OFDMA frame is set to 400. In the hotspot topology, the number of D2D pairs is fixed at 30, whereas the number of D2D pairs in the random topology changes from 20 to 70 . To verify that our proposed RACG effectively alleviates the RB usage rate and occupied rate in an OFDMA frame for D2D communication, we compare RACG and RADG in the hotspot network topology and random network topology. Table 2 summarizes the comparison between RACG and RADG on attributes of design.

4.2. Hotspot Network Topology. A hotspot network topology can be classified as high density (HD) and low density (LD). A high-density distribution indicates that the distance between D2D pairs is short, such that D2D pairs are more likely to exhibit mutual interference. By contrast, for a low- density distribution, D2D pairs are further apart and, therefore, less likely to exhibit mutual interference.

Figure 9 displays the OFDMA Frame Utilization (OFU) of high-density and low-density distributed hotspot network topologies under various bandwidth requirements for D2D pairs. We define OFU as the total number of RBs allocated to $\mathrm{D} 2 \mathrm{D}$ pairs divided by the total number of $\mathrm{RBs}$ in an OFDMA frame. In Figure 9, the red and blue lines represent the OFU of high-density and low-density distributions, respectively, whereas the solid and dashed lines represent the OFU with RACG and RADG, respectively. We observe from Figure 9 that when the number of RBs required by each D2D pair increases, the OFU of high-density distribution increases at a faster rate, whereas the OFU of low-density distribution increases at a slower rate. This is because the D2D pairs in high-density distribution exhibit more mutual interference. Hence, they have fewer reused RB chunks and the total number of RBs allocated to D2D pairs resulting in a rapid rise in OFU. In high-density distribution, the 


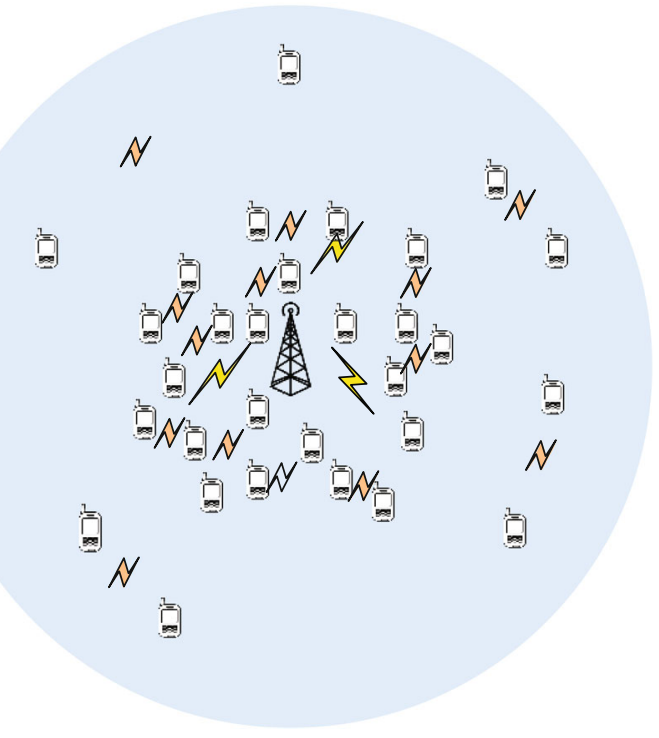

(a) Hotspot topology

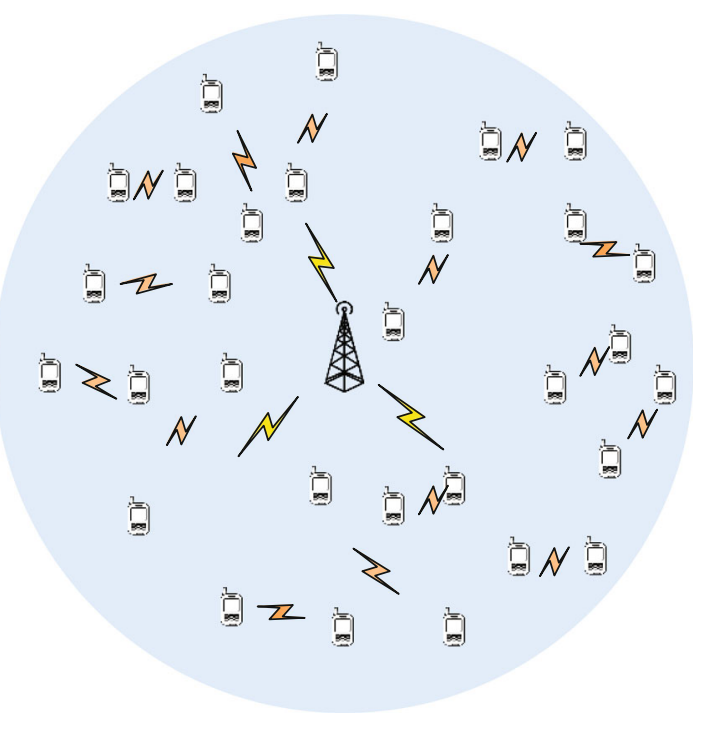

(b) Random topology

Figure 8: Two network topologies of D2D communication.

TABLE 2: Comparison between RACG and RADG.

\begin{tabular}{lcc}
\hline Attributes of design & The proposed RACG & RADG [25] \\
\hline Interference graph model & $\mathrm{v}$ & $\mathrm{v}$ \\
Identifying complete graphs & $\mathrm{v}$ & $\mathrm{x}$ \\
The degree of each vertex & $\mathrm{v}$ & $\mathrm{v}$ \\
The frequency at which each vertex appears in complete graphs & $\mathrm{v}$ & $\mathrm{x}$ \\
Various number of RB requirement & $\mathrm{v}$ & $\mathrm{v}$ \\
Allocation sequence of the vertices & $\mathrm{v}$ & $\mathrm{x}$ \\
Reusing an allocated RB chunk with the closest size & $\mathrm{x}$ \\
\hline
\end{tabular}

"v" indicates that the attributes are specified in the scheme. " $\mathrm{x}$ " indicates that the attributes are unspecified in the scheme.

difference between the OFU with RACG and RADG increases when the number of RBs required by each D2D pair increases, and this trend becomes more obvious in high-density distribution than low-density distribution. This is because RADG only avoids the level of interference but does not consider RB chunks with similar number of RBs; therefore, the OFU of RADG increases rapidly when the number of RBs required increases. Similar to RADG, RACG begins with $\mathrm{RB}$ allocation for the $\mathrm{D} 2 \mathrm{D}$ pairs in the complete graph with the largest number of vertices, that is, for those with the highest degree. Nevertheless, RACG considers three additional parameters to determine the allocation sequence for the D2D pairs, the frequency at which D2D pairs appear in complete graphs, the interference level of a D2D pair to other D2D pairs, and the number of RBs required, whereas RADG simply generates the allocation sequence at random. Besides, RACG intends allocating the reusable RB chunk that has the closest number of RBs to the number of RBs required to maximize the benefits of frequency reuse. Hence, the OFU of RACG increases at a slower rate relative to RADG.

Randomly setting the number of RBs required by each D2D pair from 31 to 35 , we vary the degree of the vertices (i.e., D2D pairs) concentrated near the eNodeB. Figure 10 presents the number of $\mathrm{RB}$ chunks used in an OFDMA frame when RACG and RADG are applied. The red and blue bars represent the number of RB chunks used by RACG and RADG, respectively. Figure 10 demonstrates that when the interference of $\mathrm{D} 2 \mathrm{D}$ pairs concentrated near the center increases or the degree number increases, the number of $\mathrm{RB}$ chunks used by RACG or RADG increases. When the degree number of the D2D pairs concentrated near the hotspot increases, the mutual interference between D2D pairs increases, resulting in most D2D pairs being unable to reuse a given RB chunk; therefore, the eNodeB has to allocate a new RB chunk to the D2D pairs. Compared with RADG, RACG achieves superior performance when the mutual interference between D2D pairs increases; this phenomenon is consistent with that observed in Figure 9.

4.3. Random Network Topology. The number of RBs required by each $\mathrm{D} 2 \mathrm{D}$ pair is randomly generated from 1 to 35 , and the total number of D2D pairs is varied from 20 to 70 . Figure 11 presents the OFU versus the total number of $\mathrm{D} 2 \mathrm{D}$ pairs in the random network topology. The red and blue lines represent the OFU with RACG and RADG, respectively. Figure 11 indicates that when the number of D2D pairs 


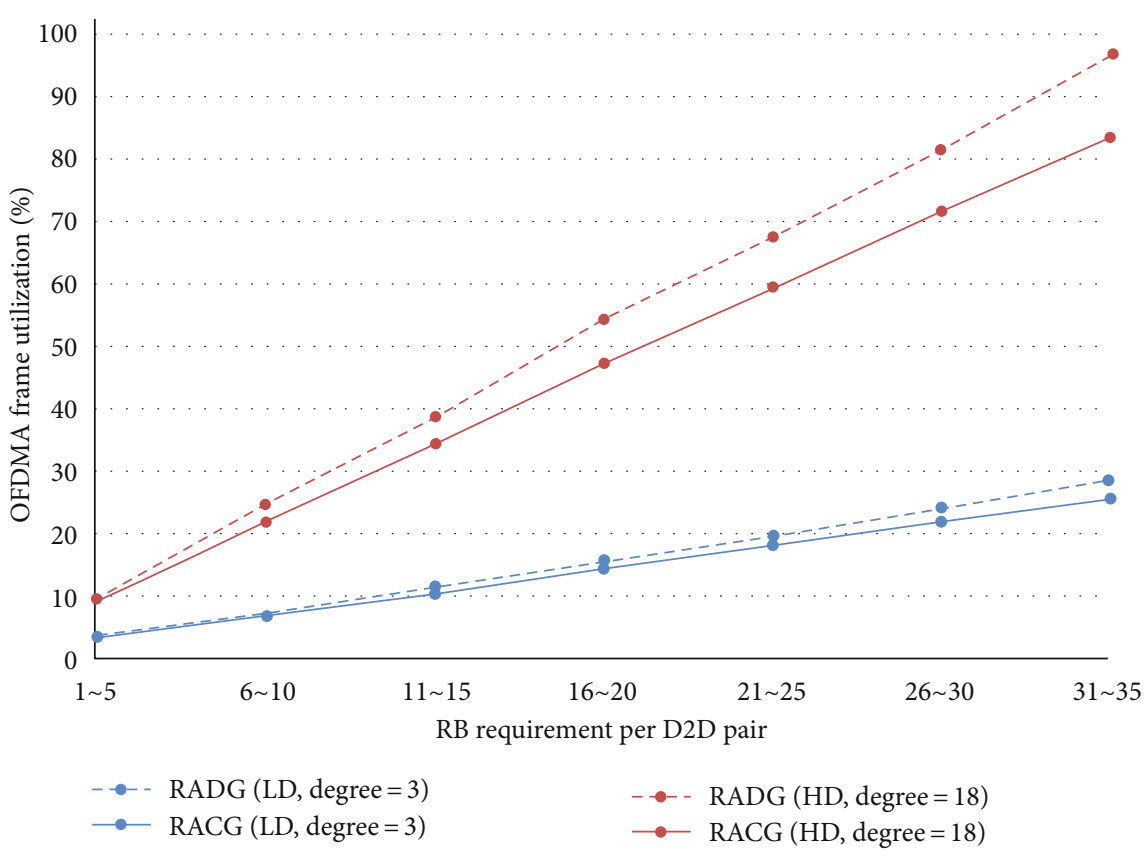

Figure 9: OFDMA frame utilization in the hotspot topology.

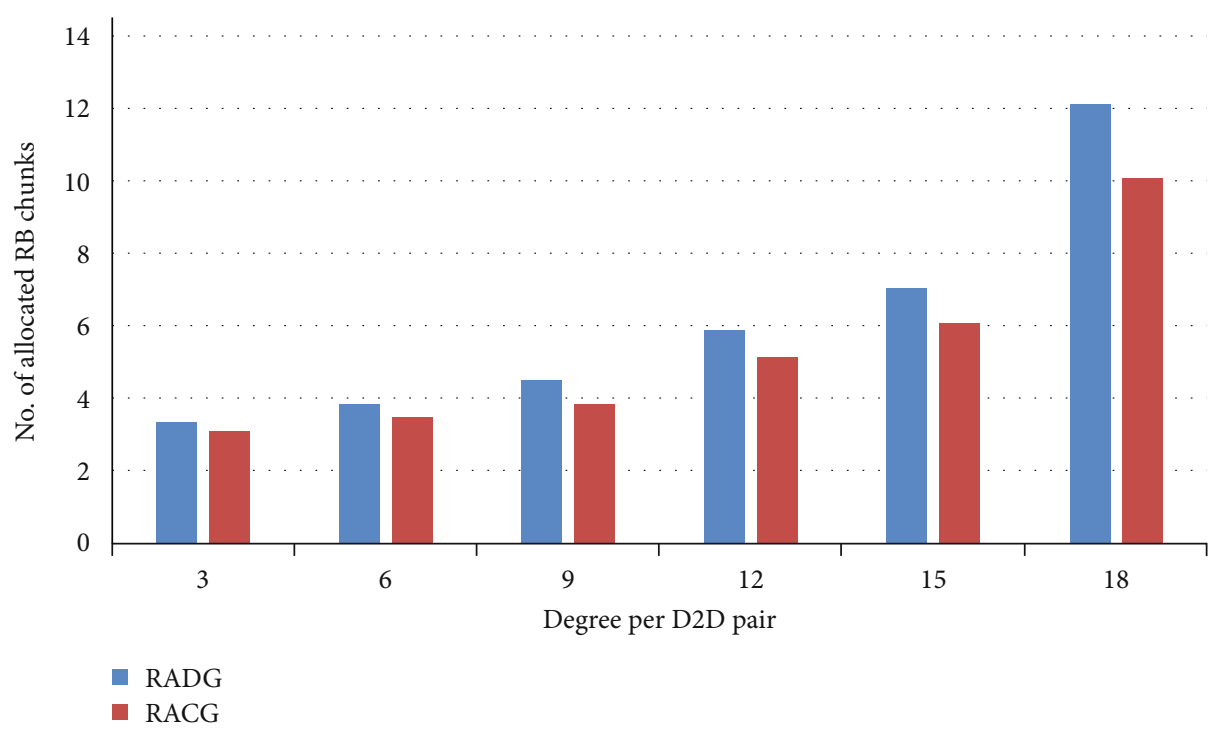

FIGURE 10: Number of allocated RB chunks in the hotspot topology.

increases, the OFU with either of RACG and RADG increases. As noted in Section 4.2, the likelihood of D2D pairs mutually interfering with each other increases when the number of D2D pairs increases. Besides, RACG considers more parameters than RADG while doing RB allocation. As a result, the OFU with RACG is lower than that with RADG. However, when the total number of $\mathrm{D} 2 \mathrm{D}$ pairs reaches 70 , almost all the RBs in an OFDMA frame are used. Hence, the OFU with either of RACG and RADG approaches $100 \%$, and the two schemes do not exhibit a difference.

With the same simulation scenario as Figure 11, Figure 12 presents the number of RB chunks used in an OFDMA frame when RACG and RADG are applied. The red and blue bars represent the number of $\mathrm{RB}$ chunks used by RACG and RADG, respectively. Figure 12 indicates that the number of RB chunks used by RACG or RADG increases when the number of $\mathrm{D} 2 \mathrm{D}$ pairs increases. The number of RB chunks used by RACG is lower than that used by RADG due to the same reason elaborated above. Referring to Figure 11, when the total number of D2D pairs reaches 70, almost all the RBs in an OFDMA frame are used. Therefore, the difference between the number of RB chunks used by RACG and RADG is extremely small.

4.4. Complexity Analysis. The time complexity of RACG is divided into five phases. In phase $1, n$ D2D pairs are mapped 


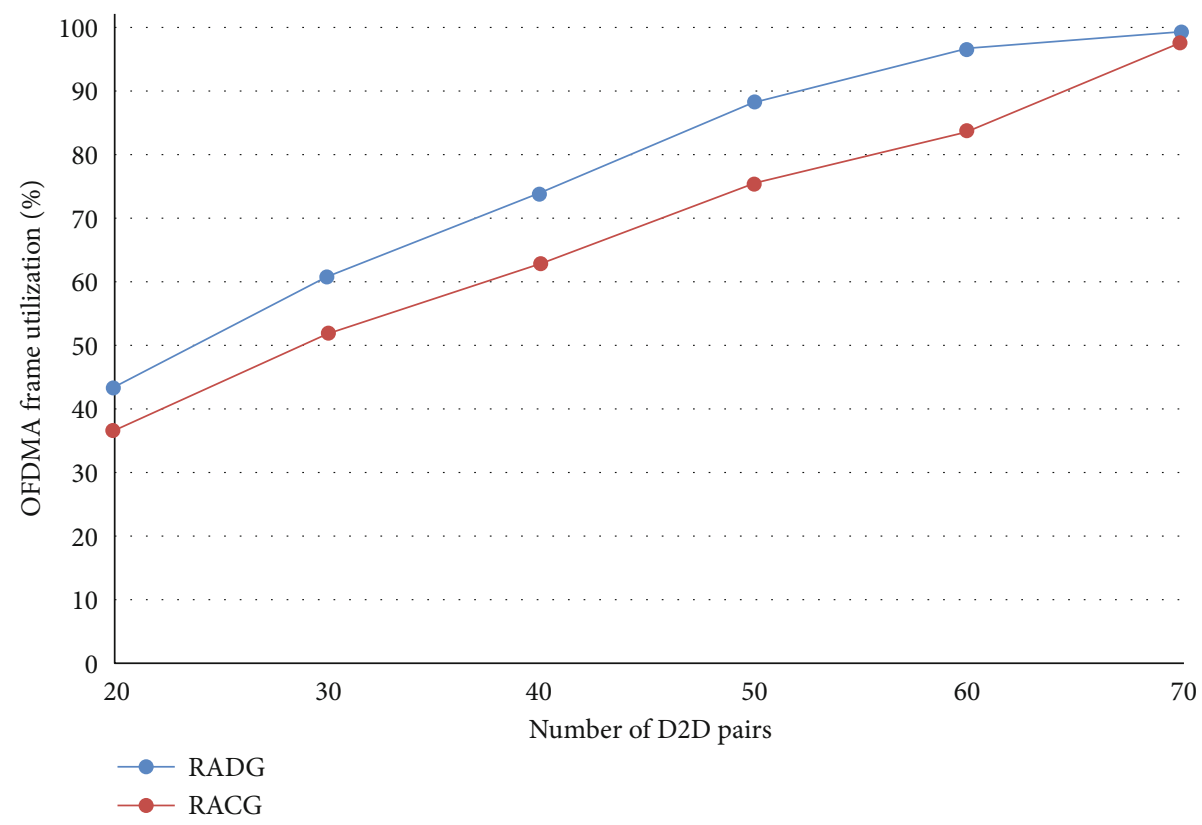

FIgURE 11: OFDMA frame utilization in the random topology.

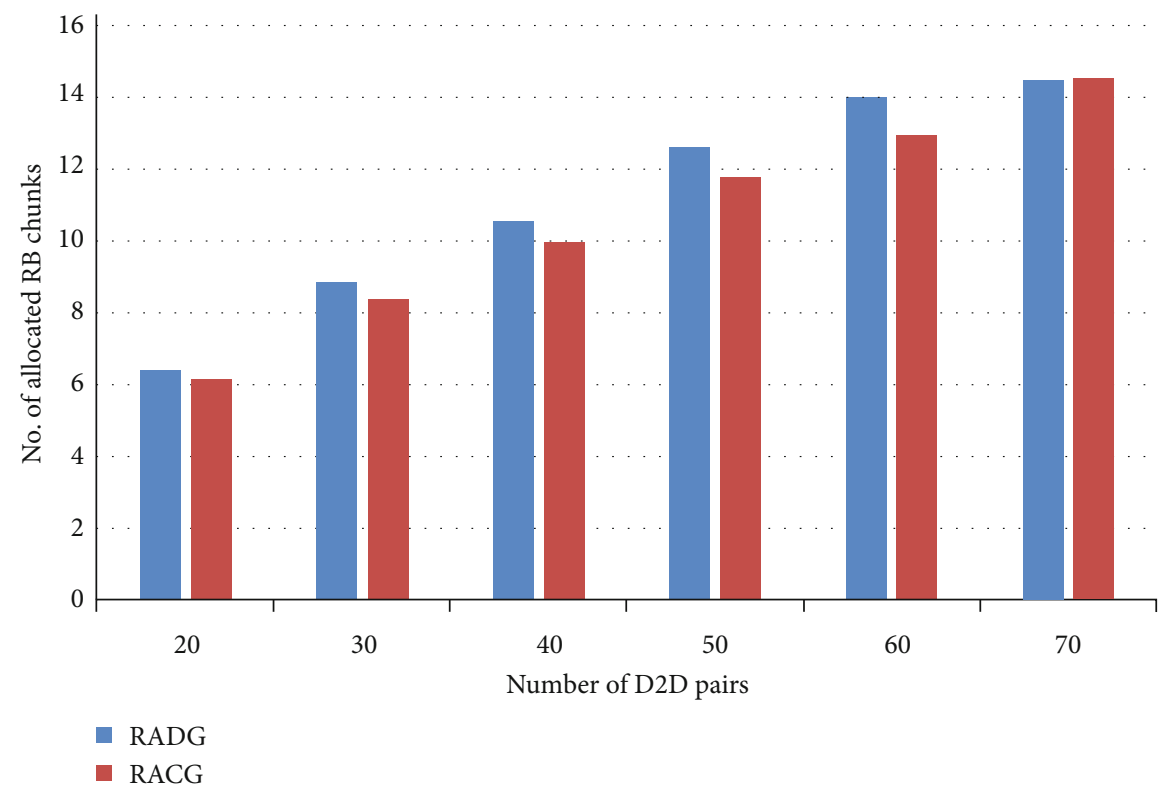

FIGURE 12: Number of RB chunks used in the random topology.

to an interference graph model. In phase 2, the number of complete graphs that each vertex belongs to is identified. In phase 3, all elements of a row in the matrix $M K_{m}$ are summed, and each vertex from the largest to smallest is ranked according to its degree. In phase 4, each vertex from the largest to smallest is ranked according to the number of RBs it required. During the final phase, the number of RBs required by each vertex is used for identifying and reusing the RB chunk that corresponds most closely to the number of RBs required by the vertex. In phase 1, the interferences between any two of D2D pairs are inputted into a twodimensional adjacency matrix; hence, the time complexity for this phase is $O\left(n^{2}\right)$. In phase 2, the Bron-Kerbosch algo- rithm is applied to identify all the complete graphs in the interference graph. Because the identification of complete graphs is an NP-complete problem, the method proposed by Moon and Moser [35] is applied to group all $n$ vertices into groups of three to form triangles. A total of $n / 3$ triangles are formed, and in each triangle, one or two vertices can form additional triangles with one or two vertices from another triangle. Therefore, the time complexity for identifying the maximum number of complete graphs is $O\left(3^{n / 3}\right)$. In phase 3 , we determine the degree of each vertex with the corresponding adjacency matrix $A$, and we rank them from the largest to smallest according to their degree number; the time complexity for this phase is $O\left(n^{2}\right)$. In phase 4 , we rank 
all the vertices from the largest to smallest according to the number of RBs they required; the time complexity for this phase is also $O\left(n^{2}\right)$. In the final phase, we use the RBs required by each vertex to select and reuse the $\mathrm{RB}$ chunk that corresponds most closely to the number of RBs required; the time complexity for this phase is $O\left(n^{2}\right)$. Thus, the time complexity of RACG is $O\left(3^{n / 3}+4 n^{2}\right)$.

On the other hand, the time complexity of RADG is divided into four phases. In phase $1, n$ D2D pairs are mapped to an interference graph model; the time complexity for this phase is $O\left(n^{2}\right)$. In phase 2, each row in the adjacency matrix $A$ is summed to obtain the degree of each vertex, and all the vertices from the largest to smallest are ranked according to their degree number; the time complexity for this phase is $O\left(n^{2}\right)$. In phase 3, all the vertices are ranked from the largest to smallest according to the number of $\mathrm{RBs}$ they required; the time complexity for this phase is $O\left(n^{2}\right)$. During the final phase, one of RB chunks is randomly selected and reused to meet the $\mathrm{RB}$ requirement of each vertex; the time complexity for this phase is $O(n)$. Thus, the time complexity of RADG is $O\left(n+3 n^{2}\right)$. Therefore, the RACG proposed in the paper enables the effective usage of RBs but at the cost of more computational time.

\section{Conclusions}

This paper proposes a heuristic $\mathrm{RB}$ allocation mechanism using complete graphs (abbreviated to RACG) for D2D communication in LTE-A and beyond mobile communication networks. RACG not only can reduce the cochannel interference but also can enhance the overall system capacity. First, RACG maps the interference between any two of D2D pairs to an interference graph model and identifies all complete graphs in the model. Next, it begins with RB allocation to the D2D pairs in the largest complete graph, and let the $\mathrm{D} 2 \mathrm{D}$ pairs with similar $\mathrm{RB}$ requirements reuse the same RB chunks if no mutual interference is present. The $\mathrm{RB}$ allocation sequence is determined according to three additional parameters, namely, the number of times D2D pairs appear in different complete graphs, the interference effect of a D2D pair on other D2D pairs, and the number of $\mathrm{RBs}$ required by a $\mathrm{D} 2 \mathrm{D}$ pair. We compare the $\mathrm{OFU}$ and number of RB chunks allocated by RACG and RADG via numerical simulations. The results reveal that the difference between RACG and RADG in the OFU increases when the interference among the D2D pairs concentrated at the center increases in the hotspot network topology. The difference between RACG and RADG in the OFU also increases when the total number of D2D pairs increases in the random network topology. On the other hand, the number of $\mathrm{RB}$ chunks used by RACG is $15 \%$ less than the number of RB chunks used by RADG in the hotspot and random network topologies. Focusing on enhancing the overall system capacity of all communication modes, we are now investigating $\mathrm{RB}$ allocation schemes by including cellular UE, which are allowed to directly connect to eNodeB through unused RBs or the RBs used by D2D pairs that do not exhibit mutual interference.

\section{Data Availability}

The MATLAB data used to support the findings of this study are available from the corresponding author upon request.

\section{Conflicts of Interest}

The authors declare that they have no conflicts of interest.

\section{Acknowledgments}

This research was supported in part by the Taiwan Ministry of Science and Technology under the Grant Nos. MOST 109-2221-E-110-043 and MOST 110-2221-E-158-001.

\section{References}

[1] F. Jameel, Z. Hamid, F. Jabeen, S. Zeadally, and M. A. Javed, “A survey of device-to-device communications: research issues and challenges," IEEE Communications Surveys \& Tutorials, vol. 20, no. 3, pp. 2133-2168, 2018.

[2] P. K. Malik, D. S. Wadhwa, and J. S. Khinda, "A survey of device to device and cooperative communication for the future cellular networks," International Journal of Wireless Information Networks, vol. 27, no. 3, pp. 411-432, 2020.

[3] "Evolved universal terrestrial radio access (E-UTRA); physical channels and modulation," 3GPP TS 36.211, ver. 12.7.0, 2015.

[4] "Evolved universal terrestrial radio access (E-UTRA) and evolved universal terrestrial radio access network," 3GPP TS 36.300, ver. 13.3.0, 2016.

[5] S. Huang, B. Liang, and J. Li, "Distributed interference and delay aware design for D2D communication in large wireless networks with adaptive interference estimation," IEEE Trans. Wireless Communications, vol. 16, no. 6, pp. 3924-3939, 2017.

[6] J. Kim, T. Kim, J. Noh, and S. Cho, "Fractional frequency reuse scheme for device to device communication underlaying cellular on wireless multimedia sensor networks," Sensors, vol. 18, no. 8, p. 2661, 2018.

[7] F. Wang, Y. Li, Z. Wang, and Z. Yang, "Social-communityaware resource allocation for D2D communications underlaying cellular networks," IEEE Transactions on Vehicular Technology, vol. 65, no. 5, pp. 3628-3640, 2016.

[8] H. Wu, L. Wang, T. Svensson, and Z. Han, "Resource allocation for wireless caching in socially-enabled D2D communications," in 2016 IEEE International Conference on Communications, pp. 1-6, Kuala Lumpur, Malaysia, 2016.

[9] D. Wu, Y. Xu, and Q. Wu, "Resource allocation for D2D wireless networks with asymmetric social weighted graph," IEEE Communications Letters, vol. 21, no. 9, pp. 2085-2088, 2017.

[10] Z. Zhou, C. Gao, and C. Xu, "Joint peer discovery and resource allocation for social-aware D2D communications: a matching approach," in 2016 IEEE International Conference on Communication Systems, pp. 1-6, Shenzhen, China, 2016.

[11] M. Nitti, G. A. Stelea, V. Popescu, and M. Fadda, "When social networks meet D2D communications: a survey," Sensors, vol. 19, no. 2, p. 396, 2019.

[12] Y. P. Llerena and P. R. L. Gondim, "Social-aware spectrum sharing for D2D communication by artificial bee colony optimization," Computer Networks, vol. 183, no. 1, article 107581, 2020. 
[13] X. Liu, X. Li, and C. Liu, "Position-based mode selection and resource allocation for D2D communications underlaying cellular networks," in 2017 International Conference on Wireless Communications and Signal Processing, pp. 1-6, Nanjing, China, 2017.

[14] F. Chiti, D. D. Giacomo, R. Fantacci, and L. Pierucci, "Interference aware approach for D2D communications," in 2016 IEEE International Conferences on Communications, pp. 1-6, Kuala Lumpur, Malaysia, 2016.

[15] M. C. Lucas-Estan and J. Gozalvez, "Distance-based radio resource allocation for device to device communications," in 2017 IEEE 85th Vehicular Technology Conference, pp. 1-6, Sydney, Australia, 2017.

[16] Z. Li, S. Chen, and C. Guo, "Location-aware hypergraph coloring based spectrum allocation for D2D communication," in 2018 15th International Symposium on Wireless Communication Systems, pp. 1-6, Lisbon, Portugal, 2018.

[17] S. Selmi and R. Bouallègue, "Interference and power management algorithm for D2D communications underlay $5 \mathrm{G}$ cellular network," in 2019 International Conference on Wireless and Mobile Computing, Networking and Communication, pp. 1-8, Barcelona, Spain, 2019.

[18] M. Rim and E. Go, "Relay positions considering interference from other sub-channels in OFDMA-based D2D groupcasting systems," Sensors, vol. 19, no. 6, p. 1374, 2019.

[19] W. Lai, Y. Wang, H. Lin, and J. Li, "Efficient resource allocation and power control for LTE-A D2D communication with pure D2D model," IEEE Transactions on Vehicular Technology, vol. 69, no. 3, pp. 3202-3216, 2020.

[20] X. Chen, R. Q. Hu, J. Jeon, and G. Wu, "Optimal resource allocation and mode selection for D2D communication underlaying cellular networks," in 2015 IEEE Global Communications Conference, pp. 1-6, San Diego, USA, 2015.

[21] B. Yu and Q. Zhu, "A QoS-based resource allocation algorithm for D2D communication underlaying cellular networks," in 2016 Sixth International Conference on Information Science and Technology, pp. 406-410, Dalian, China, 2016.

[22] C. Liu and J. Zheng, "A QoS-aware resource allocation algorithm for device-to-device communication underlaying cellular networks," in 2017 IEEE 85th Vehicular Technology Conference, pp. 1-5, Sydney, Australia, 2017.

[23] P. Khuntia and R. Hazra, "QOS aware channel and power allocation scheme for D2D enabled cellular networks," Telecommunication Systems: Modelling, Analysis, Design and Management, vol. 72, no. 4, pp. 543-554, 2019.

[24] A. M. Waswa, S. Mwanje, J. Mueckenheim, and A. MitscheleThiel, "QoS-aware spectrum sharing for D2D communication in cellular networks," in 2020 European Conference on Networks and Communications, pp. 94-99, Dubrovnik, Croatia, June 2020.

[25] C. Lee, S. Min, and J. S. Shin, "Resource allocation for deviceto-device communications based on graph-coloring," in 2015 International Symposium on Intelligent Signal Processing and Communication Systems, pp. 451-455, Nusa Dua, Indonesia, 2015.

[26] T. Yang, R. Zhang, X. Cheng, and L. Yang, "Graph coloring based resource sharing (GCRS) scheme for D2D communications underlaying full-duplex cellular networks," IEEE Trans. Vehicular Technology, vol. 66, no. 8, pp. 7506-7517, 2017.

[27] Y. Li, M. C. Gursoy, S. Velipasalar, and J. Tang, "Joint mode selection and resource allocation for D2D communications via vertex coloring," in 2017 IEEE Global Communications Conference, pp. 1-6, Singapore, 2017.

[28] A. Pratap and R. Misra, "Resource sharing in D2D communication underlaying cellular LTE-A networks," in 2015 International Conference on Advances in Computing, Communications and Informatics, pp. 103-109, Kochi, India, 2015.

[29] R. M. Alsharfa, S. L. Mohammed, S. K. Gharghan, I. Khan, and B. J. Choi, "Cellular-D2D resource allocation algorithm based on user fairness," Electronics, vol. 9, no. 3, p. 386, 2020.

[30] K. Zhao, M. Sheng, J. Liu et al., "Graph-based joint mode selection and resource allocation scheme for D2D and cellular hybrid network using SCMA," in 2016 International Conference on Wireless Communications \& Signal Processing, pp. 15, Yangzhou, China, 2016.

[31] X. Cai, J. Zheng, and Y. Zhang, "A graph-coloring based resource allocation algorithm for D2D communication in cellular networks," in 2015 IEEE International Conference on Communications, pp. 5429-5434, London, UK, 2015.

[32] C. Bron and J. Kerbosch, "Algorithm 457: finding all cliques of an undirected graph," Communications of the ACM, vol. 16, no. 9, pp. 575-577, 1973.

[33] R. P. Grimaldi, Discrete and Combinatorial Mathematics, Pearson Education, India, 2004.

[34] MATLABhttps://www.mathworks.com/products/matlab .html.

[35] J. W. Moon and L. Moser, "On cliques in graphs," Israel Journal of Mathematics, vol. 3, no. 1, pp. 23-28, 1965. 